\title{
Das Digital Canvas: Ein Instrument zur Konzeption digitaler Geschäftsmodelle
}

\author{
Ricarda Schlimbach (D) Reza Asghari
}

Eingegangen: 30. März 2020 / Angenommen: 6. Mai 2020 / Online publiziert: 15. Mai 2020

(C) Der/die Autor(en) 2020

Zusammenfassung Der Megatrend der Digitalisierung verändert gravierend die Architektur von Geschäftsmodellen und erfordert diesbezüglich überarbeitete Instrumente zur konzeptionellen Unterstützung. Der Kern dieses Beitrags ist die Vorstellung des Digital Canvas, einer auf digitale Geschäftsmodelle spezifizierten Alternative zum weit verbreiteten generischen Business Model Canvas zur Geschäftsmodellkonzeption. Methodisch wurden zur Ermittlung der Anforderungen an dieses neue Artefakt eine Online-Befragung mit den Teilnehmern einer Entrepreneurship Summer School und 16 semi-strukturierte Experteninterviews ausgewertet und mit Erkenntnissen der Literatur trianguliert. Basierend auf den identifizierten Schwachstellen des weit verbreiteten Business Model Canvas im Digitalkontext und synthetisierten Anforderungen an ein digitalspezifisches Visualisierungsinstrument entsteht das Digital Canvas als Lösungsvorschlag. Dieses besteht aus neun Bausteinen, deren Inhalte kompakt vorgestellt werden und durch einen bereitgestellten Leitfaden bei der systematischen Erarbeitung unterstützen. Die Potentiale und Limitationen des an das digital transformierende Ökosystem angepasste Canvas werden diskutiert. Im Ergebnis steht ein praxisnahes Hilfsmittel zur Ausgestaltung digitaler Geschäftsmodelle bereit, welches sich für den Einsatz in der Lehre als auch für die praktische Nutzung durch Entrepreneure eignet.

Schlüsselwörter Business Model Canvas · Digital Canvas · Digitales Geschäftsmodell · Geschäftsmodellvisualisierung · Digitale Transformation

R. Schlimbach $(\bowtie) \cdot$ R. Asghari

Entrepreneurship Hub, TU Braunschweig, Rebenring 33, 38106 Braunschweig, Deutschland

E-Mail: ricarda.schlimbach@tu-bs.de 


\title{
The Digital Canvas: An Instrument for the Creation of Digital Business Models
}

\begin{abstract}
The megatrend of digitization is changing the architecture of business models and requires revised instruments for conceptual support. The core of this paper is the presentation of the Digital Canvas, an alternative to the widespread generic Business Model Canvas for business model conception, which is specified on digital business models. Methodologically, an online survey with participants of an Entrepreneurship Summer School and 16 semi-structured expert interviews were evaluated and triangulated with findings from literature to determine the requirements for this new artefact. Based on the identified weaknesses of the widespread Business Model Canvas in the digital context and synthesized requirements for a digitally specific visualization tool, the digital canvas is developed as a proposed solution. It consists of nine building blocks, whose contents are presented in a compact form and support the systematic development with a guide provided. The potentials and limitations of the canvas adapted to the digital transforming ecosystem are discussed. The result is a practical tool for the design of digital business models, which is suitable for use in teaching as well as for practical use by entrepreneurs.
\end{abstract}

Keywords Business Model Canvas · Digital Canvas · Digital Business Model · Business Model Visualization · Digital Transformation

\section{Einleitung}

Die Digitalisierung katalysiert als bislang bedeutendster Megatrend des 21. Jahrhunderts die Notwendigkeit der Innovation bislang analog geprägter Geschäftsmodelle (Hamidian und Kraijo 2013). Sie löst einen dynamischen Wandlungsprozess am Markt aus und verändert rasant die herkömmlichen Geschäftsmodelle (GM). GM etablierter Großunternehmen werden von jungen Start-Ups disruptiv zerstört und durch marktfähigere und kundenfreundlichere ersetzt - beispielsweise verdrängte Netflix durch ein Video-on-Demand-Angebot den damaligen Branchenriesen Blockbuster vom Markt und AirBnB disruptierte durch das Plattformgeschäftsmodell die Beherbergungsbranche ohne ein einziges Bett zu besitzen (Kreutzer und Land 2016). Dieser Umstand stellt Unternehmen vor große Herausforderungen und erfordert neue effektive Instrumente, welche an das hochdynamische und zunehmend digitalisierte Wirtschaftsumfeld angepasst sind (Wirtz et al. 2016). Weil jedoch angepasste Instrumente fehlen, wird weiterhin auf das Konzept des Business Model Canvas (BMC) zur Gestaltung und Visualisierung von GM zurückgegriffen.

Das von Osterwalder und Pigneur (2010) entwickelte BMC reduziert ein GM auf die in Abb. 1 dargestellten neun grundlegenden Bausteine.

Der große Erfolg dieses konzeptuellen Instruments in Wissenschaft und Praxis wird durch die hohe Zitationshäufigkeit, dessen Übersetzung in 30 Sprachen und seine Integration in das Lehrkonzept von über 250 Universitäten deutlich (Stenn 2017). Das BMC unterstützt bei der Reduktion des komplexen Geschäftsmodellkonstrukts 


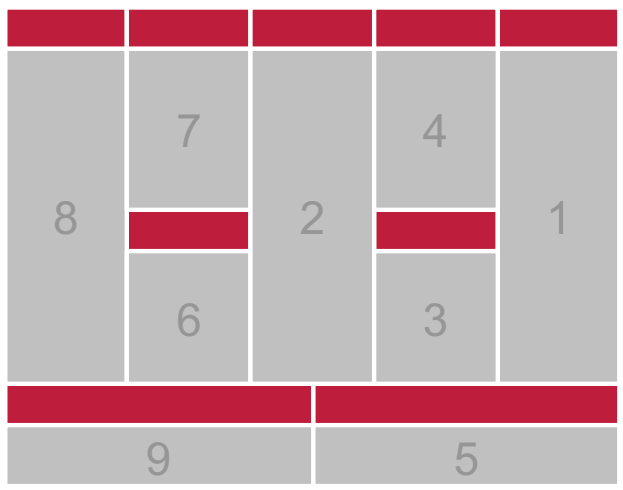

1. Kundensegmente

2. Wertangebot

3. Kanäle

4. Kundenbeziehung

5. Einnahmequellen

6. Schlüsselressourcen

7. Schlüsselaktivitäten

8. Schlüsselpartner

9. Kostenstruktur

Abb. 1 Das Business Model Canvas nach Osterwalder und Pigneur 2010

auf die wesentlichen Bausteine und regt zur Diskussion, Innovation und Reflektion an (Trimi und Berbegal-Mirabent 2012).

Nichtsdestotrotz fehlt dem BMC eine Adaption an die neuen Rahmenbedingungen der sich zunehmend digital transformierenden Ökosysteme (Simmert et al. 2014). Dazu zählen insbesondere die zumeist datengetriebene gemeinsame Wertschöpfung zwischen Anbieter und Nutzer (Pfeiffer 2018), die Verschiebung einer produktzentrierten hin zu einer dienstleistungsorientierten Logik (Vargo und Lusch 2008), steigende Kundenzentrierung als Markterwartung (Reinartz 2018), Daten als Treiber neuer Geschäftschancen (Iansiti und Lakhani 2014) und die organisationale Anpassungsfähigkeiten an die sich transformierende Umwelt als neuer wettbewerbsentscheidender Faktor (Downs und Velamuri 2018).

Im Kontext dieser Entwicklungen werden in diesem Beitrag Schwachstellen des BMC aus der Anwenderperspektive untersucht. Ferner werden praxisnah Anforderungen an ein zeitgemäßes digitalspezifisches Canvas abgeleitet und in das neukonzipierte Digital Canvas (DC) als Lösungsvorschlag überführt.

\section{Methodisches Vorgehen}

Die Forschungsergebnisse dieser Studie wurden durch die Triangulation von Daten aus der Durchführung einer Online-Umfrage sowie durch qualitative Experteninterviews, angereichert durch Erkenntnisse aus der Literatur gewonnen. Befragt wurden Studierende des Entrepreneurships und erfahrene Entrepreneure, weil diese Gruppen zu den Hauptnutzern von Hilfsmitteln zur Geschäftsmodellkonzeption zählen und ihre Erfahrungen und Anforderungen daher von besonderer Bedeutung für das Forschungsvorhaben sind.

\subsection{Online-Befragung}

Im Rahmen der im September 2018 durchgeführten Entrepreneurship Summer School an der TU Braunschweig wurde den 60 Teilnehmern das BMC detailliert 
vorgestellt. Die Teilnehmer erarbeiteten in Kleingruppen eigene GM und wurden ermutigt, das BMC im Zuge dessen einzusetzen. Im Laufe der einwöchigen Veranstaltung wurden die Studierenden gebeten, an einer Online-Umfrage teilzunehmen, um (u. a.) Angaben zu ihren Erfahrungen mit dem BMC abzugeben und den Sollzustand eines verbesserten Werkzeugs zu skizzieren. 37 Teilnehmer beantworteten den vollstandardisierten Online-Fragebogen. Die Beobachtung aller Studierenden während der Konzeptionsphase unterstützte die Datentriangulation und Interpretation der Ergebnisse.

\subsection{Experteninterviews}

Im zweiten Teil der empirischen Erhebung wurden teilstandardisierte Einzelinterviews mit Experten als grundlegende Quelle zur Erfassung von Anforderungen an ein dynamikrobustes Instrument durchgeführt. Dabei wurde auf die Auswahlkriterien nach Meuser und Nagel (2009) zurückgegriffen, um interdisziplinär und aus Detailwissen und übergeordnetem Wissen fallspezifische Informationen zu sammeln. Teilstandardisierte Interviews ermöglichten genügend Raum für Flexibilität in Hinblick auf Zwischenfragen, abgeänderte oder ergänzte Leitfragen bei gleichzeitiger Vergleichbarkeit der Ergebnisse (Döring und Bortz 2016). Die folgende Forschungsfrage leitete die Interviews: Welche Schwachstellen weist das BMC im Praxiseinsatz auf und welche Anforderungen bestehen an ein überarbeitetes Canvas hinsichtlich digitaler Geschäftsmodelle?

Insgesamt konnten 16 deutschsprachige Experten aus 16 verschiedenen Unternehmen im Frühjahr 2019 zur Teilnahme an der Studie gewonnen werden. 12 Experten hatten jeweils schon selbst erfolgreich mehrere Unternehmen gegründet und geführt. Jedem Experten konnte dabei mindestens ein digitales GM zugeordnet werden und 8 GM mussten aufgrund der digitalen Transformation bereits innoviert werden. Die übrigen 4 Experten bekleiden leitende Funktionen von Innovationsabteilungen großer Unternehmen mit dem Fokus der digitalen Geschäftsmodellinnovation. Die durchschnittlich ca. halbstündigen Experteninterviews wurden anschließend mit Hilfe der Software amberscript vollständig transkribiert und durch eine detaillierte Inhaltsanalyse nach Mayring (2002) ausgewertet.

\section{Forschungsergebnisse}

Im Folgenden werden Schwachstellen am BMC abgeleitet aus den Ergebnissen der qualitativen und quantitativen Untersuchung identifiziert und Anforderungen an eine dem digital transformierenden Umfeld angepasste Visualisierungsgrundlage herausgearbeitet. Resultierend daraus wird das neukonzeptionierte Digital Canvas als Lösungsvorschlag vorgestellt.

\subsection{Studienergebnisse}

Von den 60 Teilnehmern der Online-Befragung füllten 37 den Fragebogen vollständig aus. 16,2\% der Teilnehmer hatten schon ein eigenes Unternehmen gegründet, 
weitere $54,1 \%$ setzten sich nach eigenen Angaben ernsthaft mit dem Gedanken auseinander selbst zu gründen, wohingegen die übrigen $29,7 \%$ bislang kein reales Gründungsvorhaben verfolgten.

Die Interviewpartner der Experteninterviews kamen aus den Branchen Medizintechnik, Software, Consulting, Automobil, Lebensmittteltechnik, E-Commerce und Logistik. 13 der 16 Teilnehmer waren männlich und alle Entrepreneure haben den Stammsitz ihres Unternehmens in Deutschland.

\subsubsection{Identifizierte Schwachstellen des BMC}

Die Praxisrelevanz eines überarbeiteten Canvas konnte in den Studien nachgewiesen werden. 55\% der Befragungsteilnehmer geben an, sich mit der Geschäftsmodellkonzeption überfordert zu fühlen und wünschen sich stärkere Unterstützung. Knapp $65 \%$ geben an die Geschäftsmodellkonzeption in der Praxis eher zufällig umzusetzen, weil ein unterstützendes Instrument fehlt.

Die Umfrageteilnehmer schätzten funktionale Eigenschaften des BMC auf einer Likert-Skala ein (siehe unten). Für jedes abgefragte Attribut wurde entsprechend dem Zustimmungsgrad auf der siebenstufigen Skala ein Gesamtscore errechnet, welcher Werte zwischen 0 und 100 annehmen kann und im Fließtext in Klammern hinter der Eigenschaft angegeben wird. Aufgrund der äquidistant gewählten Skalen errechnet sich der Gesamtscore der Antwortgrundgesamtheit $N$ bei $n$ möglichen Antwortausprägungen und $\mathrm{x}$ absoluten Nennungen in der jeweiligen Antwortkategorie i nach der folgenden Formel:

$$
\text { Gesamtscore }=\sum_{i=1}^{n} \frac{i-1}{n-1} \cdot x_{i} \cdot \frac{1}{N} \text { mit } N=\sum_{i}^{n} x_{i}
$$

Der errechnete Gesamtscore gibt Auskunft über die Schwachstellen und Potentiale des BMC. Die mit der höchsten Zustimmung bewerteten Ausprägungen des BMC umfassen dessen leichte Verständlichkeit und Handhabung $(79,6)$, die Unterstützung bei der systematischen Entwicklung eines GM $(77,8)$ und seine Praxisorientierung $(76,9)$. Unter den Eigenschaften mit dem geringsten Erfüllungsgrad finden sind hingegen die Berücksichtigung der Rahmenbedingungen des Ökosystems $(48,1)$, Spaß bei der Anwendung $(66,7)$ und die Sensibilisierung für verschiedene Blickwinkel eines Geschäftsmodells $(67,6)$.

Auch alle befragten Experten der qualitativen Studie hatten schon Erfahrungen mit dem BMC gesammelt und äußerten ähnliche Kritikpunkte. Dazu zählt das dogmatische Vorgehen: „Man ist so geblendet von dem Tool, dass man lieber das Canvas ausfüllt, als sich die richtig wichtigen Fragen zu stellen" und die fehlende Thematisierung des angebotenen Mehrwerts. Ein Experte bemerkt, das BMC sollte für die Ideenfindung nur für diejenigen Komponenten genutzt werden, die ,offline passieren". Für die in seinem Unternehmen überwiegend digital gestalteten GM schätzt er das BMC hingegen aufgrund fehlender Spezialisierung auf digitale Besonderheiten als wenig hilfreich ein. Die Visualisierungsgrundlage erscheint ihm ,zu statisch“. 14 der 16 Experten bemängeln, dass die Wechselwirkung mit externen Rahmenbedingungen im BMC keine Berücksichtigung finden. Genau wie in in der Online- 
Befragung wird von mehreren Experten die fehlende Kundenzentrierung im BMC bemängelt, denn ,wir können nur erfolgreich sein, wenn wir uns stärker an die Bedürfnisse der Kunden anpassen.“

\subsubsection{Anforderungen an ein zeitgemäßes Canvas}

In beiden Studien wurde ein Sollzustand umrissen, um ein zeitgemäßes Canvas besser zu charakterisieren.

Ausgehend von in der Literatur identifizierten Schwachstellen des BMC (Ching und Fauvel 2013; Wagner et al. 2015) wurden mögliche Anforderungen an ein verbessertes Canvas definiert und in den Fragebogen integriert.

Wenn man alle Antworten der 37 Online-Umfrageteilnehmer, die mindestens einen Gesamtscore von 80 erreichen, absteigend ordnet, ergibt sich dabei die in Abb. 2 dargestellte Reihenfolge der praxisrelevanten Anforderungen.

Interessant ist, dass das Kriterium mit der höchsten Relevanz $(86,5)$ für die befragten Studierenden das zentrale Nutzerproblem bzw. Kundenbedürfnis zu fokussieren ist, gefolgt von der Forderung nach einem dynamischen Ansatz $(85,6)$ und dem Herausarbeiten des Nutzervorteils $(85,6)$. Genau diese Kriterien werden bislang jedoch kaum im BCM berücksichtigt und münden in der Forderung nach einer stärkeren Kundenorientierung und Dynamik.

Auch die bei der Ist-Analyse mit nur geringer Zustimmung erfüllten Kriterien des Austauschs im Team und der Beachtung von Rahmenbedingungen des Ökosystems, finden sich im oberen Quartil des erreichbaren Gesamtscores für den Soll-Zustand eines neuartigen Instruments, gehören aber gleichzeitig zu den schwach ausgeprägten Eigenschaften des BMC. Daher müssen diese Aspekte in den Lösungsvorschlag einfließen. An nächster Stelle wird ein Fokus auf Anforderungen, die sich aus der Digitalisierung ergeben $(81,5)$ gefordert. Da keiner der neun Bausteine des BMC bis-

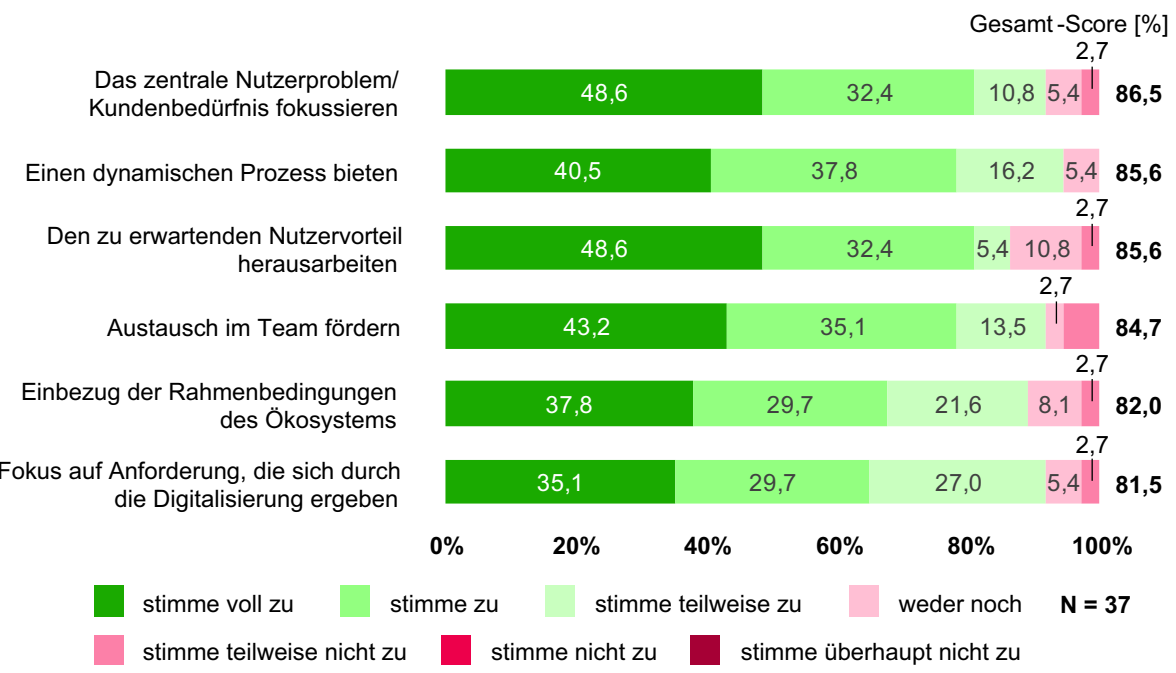

Abb. 2 Anforderungen an das Framework 
lang einen solchen Fokus verfolgt, muss dieser Aspekt unbedingt Berücksichtigung finden.

Aus der qualitativen Untersuchung konnten die folgenden Anforderungen an ein überarbeitetes Canvas synthetisiert werden:

1. Fokussierung des Kunden mit seinen herausgearbeiteten Problemen und Bedürfnissen

2. Herausarbeiten des geschaffenen Mehrwerts für den Kunden

3. Kopplung des GMs an die Rahmenbedingungen, insbesondere der technologischen Möglichkeiten und Trends, der Marktstruktur, gesellschaftlicher Charakteristika und einzuhaltenden Regularien des Zielmarktes

4. Daten als neuer fundamentaler Bausteine eines GMs

5. Integration der Komponente menschlicher Fähigkeiten als eigene Ressource

6. Hybridisierung des Leistungsangebots in physische und digitale Komponente als digitalspezifische Besonderheit und wachsende Dienstleistungsorientierung

7. Integration der Anpassungsfähigkeit der Organisationsstruktur und des GMs selbst als überlebenswichtiger Faktor

8. Bearbeitungshilfe zum Canvas, welche eine multiperspektivische Betrachtung und die dynamische Kommunikation der einzelnen Bausteine zu verschiedenen Zeitpunkten unterstützt

Übergeordnet untermauern die Äußerungen der Befragten ,aufgrund der Digitalisierung sind die Markteintrittsbarrieren viel geringer und auch Start-ups mit kaum Ressourcen können den großen Unternehmen auf einmal richtig Druck machen", dass die Größe eines Unternehmens und der Zugang zu Ressourcen immer weniger wettbewerbsentscheidend sind und viel mehr die schnelle Anpassungsfähigkeit an geänderte Umweltzustände über das Überleben am Markt bestimmen: „,adapt or die ist die Devise".

Insgesamt weicht in beiden Studien der gezeichnete Istzustand hinsichtlich des untersuchten BMC vom gewünschten Sollzustand $a b$, weswegen wie prognostiziert geschlussfolgert werden kann, dass das BMC dem hochkomplexen digital transformierenden Ökosystem nicht mehr gerecht wird und daher einer Überarbeitung bedarf. Basierend auf den identifizierten Anforderungen an ein überarbeitetes Canvas, wird im nächsten Unterabschnitt das DC als Lösungsvorschlag eingeführt.

\subsection{Das Digital Canvas als Lösungsvorschlag}

Als visualisierende Unterstützung ermöglicht das neu konzipierte DC einen komprimierten Blick auf die einzelnen im Digitalkontext relevanten Bausteine eines GMs. Das DC visualisiert durch die Positionierung seiner Komponenten deren Abhängigkeiten. Strukturell angelehnt an das BMC und inhaltlich dennoch neuartig, vereint es veränderte externe Rahmenbedingungen mit den digitalspezifischen Anforderungen an GM im komplexen Wirtschaftsumfeld. 


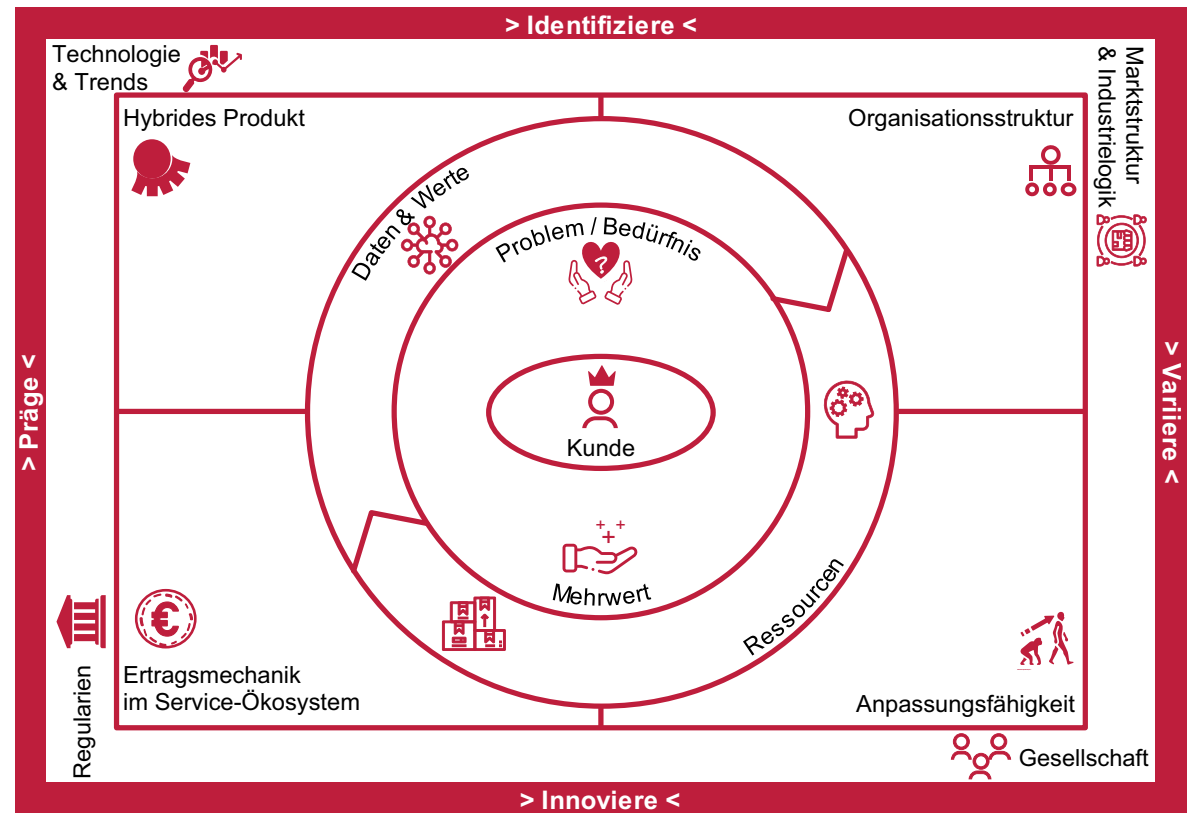

Abb. 3 Das Digital Canvas

\subsubsection{Aufbau und Bausteine}

Das DC ist in Abb. 3 mit seinen einzelnen Bausteinen illustriert.

Der Aufbau des DC ist bewusst so gewählt, dass analog der Anforderung steigender Kundenzentrierung der Kunde auch im Zentrum der Visualisierungsgrundlage steht. Den äußeren Rahmen hingegen bilden die vier Säulen Technologie \& Trends, Marktstruktur \& Industrielogik, Gesellschaft und Regularien als grundlegende Eckpfeiler einer sich wandelnden Umwelt. Somit wird der bisherigen Schwachstelle der Entkopplung des GMs von seiner Umwelt entgegengewirkt. Alle weiteren Bausteine dürfen nie losgelöst von den diesbezüglich identifizierten Umweltparametern konzipiert werden.

Der rot abgehobene Rand unterstreicht die geforderte Dynamik im Konzeptionsprozess. Das DC soll daher ausdrücklich nicht verwendet werden, um statisch eine Momentaufnahme eines GMs zu skizzieren, sondern dynamisch Zustandsänderungen abbilden, wobei veränderte Einflussparameter identifiziert, Bausteine variiert, das GM innoviert und schlussendlich das Umfeld hierdurch geprägt werden.

Im Zentrum werden an den Kunden anknüpfend dessen Probleme und Bedürfnisse identifiziert und der Mehrwert, welcher durch das GM geschaffen werden soll, festgehalten. Da es Konstellationen geben kann, in denen der zahlende Kunde nicht kongruent mit dem Nutzer des Leistungsangebotes ist, muss diesbezüglich ggf. eine Differenzierung vorgenommen werden, sodass der jeweils geschaffene Mehrwert einzeln herausgearbeitet wird. 
Ein kreisförmig umlaufender Pfeil illustriert die gemeinsame Ressourcenintegration von Anbieter und Nachfrager im Zuge der gemeinsamen Wertschöpfung, wie sie in digitalen Ökosystemen üblich ist. In einem solchen Service-Ökosystem werden nicht mehr Produkte gegen Geld getauscht, sondern verschiedene Akteure tauschen Services aus und erschaffen dadurch einen Gebrauchswert (Pfeiffer 2018). Die Symbole im DC stehen dabei für Daten, Werte, operande und operante Ressourcen. Die Positionierung dieser Ressourcenintegration ist bewusst als verbindendes Element zwischen dem Kundenbedürfnis, dem geschaffenen Mehrwert und den das GM strukturierenden Kernbausteinen gewählt worden, da die Ressourcen (insbesondere gewonnene Daten) einerseits neue Kundenbedürfnisse vorhersagen und befriedigen können und andererseits auch das Leistungsangebot selbst, die Ertragsmechanik, Anpassungsfähigkeit und komplexitätsgerechte Organisationstrukturen beeinflussen.

Das Element des hybriden Produkts dient zur mentalen Aufspaltung der physischen und digitalen Komponente des Leistungsangebots und grenzt bewusst an die Technologien und Trends der Umwelt an, weil diese Treiber Potentiale zur weiteren technischen Ausgestaltung der digitalen Dienste und des Leistungsangebots liefern.

Die Organisationsstruktur muss reaktionsfähig gestaltet sein und genügend Handlungsalternativen im komplexen Umfeld bieten. Hier können innovationsfördernde Strukturen, das Führungsleitbild, der Umgang mit Überraschungen aus dem Markt oder allgemein Maßnahmen zur Steigerung der Binnenkomplexität eingetragen werden.

Gesellschaftlicher Wandel und technologischer Fortschritt erfordern die anpassungsfähige Ausgestaltung der Wertschaffung. Insbesondere sollen Möglichkeiten zur flexiblen Anpassung einzelner Dimensionen des GMs oder der umliegenden Organisationsstruktur bzgl. potentieller neuer Anforderungen durchdacht werden.

Die Ertragsmechanik hält die konkrete Architektur der Gewinnerzielung fest und schließt die Einnahmequellen und Kostenstruktur mit ein. Dabei muss die gefundene Architektur im Einklang zu gesetzlichen Regularien stehen und mit den gesellschaftlichen Werten sowie den zur Verfügung stehenden Ressourcen harmonieren. Deshalb ist die Ertragsmechanik daran angrenzend dort angeordnet.

\subsubsection{Begleitende Leitfragen zu den einzelnen Bausteinen}

Entsprechend der Anforderung aus den Studienergebnissen, soll die Arbeit mit dem DC durch einen begleitenden Fragebogen unterstützt werden. Empfehlenswert ist daher eine systematische Bearbeitung der Leitfragen in der vorgeschlagenen Reihenfolge. Idealerweise erfolgt die Beantwortung aus verschiedenen Stakeholder-Perspektiven heraus. Dadurch können Synergien aber auch Interessenskonflikte leichter erkannt werden.

1. Rahmenbedingungen:

- Welche Technologien und Trends dominieren den Markt? Wie könnten diese in das GM integriert werden? Welche Technologien sind nützlich/hinderlich und welche Trends beflügeln/behindern das eigene GM? 
- Welche Marktstruktur dominiert (Größe, Wettbewerber, Marktform)? Gibt es ein vorherrschendes Muster der Wertschöpfung (z. B. Digitalplattform, Sharing Economy, etc.)?

- Welche gesellschaftlichen Trends und Rahmenbedingungen herrschen vor?

- Welche Regularien sind für das GM relevant und welche Auswirkungen haben diese?

2. Kunde:

- Wer wird das Leistungsangebot nutzen und wer soll dafür bezahlen? Charakterisieren Sie Ihren Kunden näher (Alter, Lebenssituation, Kaufkraft, Gewohnheiten, Erreichbarkeit, etc.).

3. Problem/Bedürfnis und Mehrwert

- Mit welchem echten Problem ist der Kunde konfrontiert? Welche offensichtlichen oder latenten Bedürfnisse hat er?

- Welchen Mehrwert bietet das GM, um auf diese identifizierten Probleme und Bedürfnisse $\mathrm{zu}$ reagieren? Welche Alternativen hat der Kunde? In welchem Verhältnis steht sein Leidensdruck zum Angebot?

4. Ressourcen

- Welche operanden, also physischen/materiellen Ressourcen sind für das GM von Bedeutung?

- Welche operanten, also immateriellen/fähigkeitsbasierten Ressourcen und Schlüsselaktivitäten sind für das GM von Bedeutung? Welche Ressourcen müssen Sie einerseits selbst einbringen und welche Ressourcen könnten durch Drittanbieter oder die Kunden selbst integriert werden?

5. Daten und Werte

- Welche Rolle spielen Daten und nicht-monetäre Werte (z. B. Reputation, Vertrauen) für Ihr GM? Welche Möglichkeiten gibt es diese sinnvoll zu nutzen, aber gleichzeitig zu schützen? Welche neuen Geschäftspotentiale ergeben sich?

6. Hybrides Produkt

- Verfügen Sie über ein Hybrides Produkt? Wie können sich die physische und die digitale Komponente ergänzen?

7. Organisationsstruktur

- Wie ist die Organisationsstruktur aufgebaut? Welche Maßnahmen helfen Ihnen dabei flexibel umstrukturieren zu können? Welches Verständnis von Zusammenarbeit, Führung und strategischer Ausrichtung verfolgen Sie?

8. Ertragsmechanik im Service-Ökosystem

- Welche Werte und Services tauschen die Akteure aus und wie kann dadurch langfristig Geld verdient werden? Welche Einnahmequellen und Kosten gibt es? Welche Zahlungsmöglichkeiten gibt es für die Kunden? Ab wann ist das GM rentabel?

9. Anpassungsfähigkeit

- Welche Bausteine können ohne großen Zeit- und Ressorucenaufwand flexibel angepasst werden? Wie könnten Geschäftsmodellmuster (Wettbewerber, andere Branche, etc.) übertragen oder kombiniert werden? Welche Möglichkeiten 
der Spezialisierung sehen Sie? Erscheint es sinnvoll einzelne Bausteine des GMs zu diversifizieren?

\section{Diskussion}

Die Ergebnisse der Studien überschneiden sich stark mit kritischen Literaturbeiträgen bezüglich des BMC. So wird auch in der Literatur fehlende Dynamik und Interaktion, genau wie die fehlende Kundenperspektive sowie die Entkopplung von sich wandelnden Rahmenbedingungen kritisiert (Wagner et al. 2015; Ching und Fauvel 2013; Coes 2014; Simmert et al. 2014). Das DC reagiert auf diese Schwachstellen, indem es den Kunden fokussiert, Wechselwirkungen mit technologischen, gesellschaftlichen, regulativen und marktspezifischen Rahmenbedingungen berücksichtigt, einen dynamische Betrachtung fördert und neue digitale Bausteine der Wertschöpfung (Daten, hybride Produkte, gemeinsame Ressourcenintegration) inkludiert.

Der dank Datentriangulation fundiert erarbeitete Lösungsvorschlag konnte schon im Rahmen der Entrepreneurship Spring School 2020 zur Geschäftsmodellkonzeption erstmalig eingesetzt werden.

Erste Reflektionen führen zu der Erkenntnis, dass das DC eine leicht verständliche Visualisierungsstütze bietet und die Diskussion von digitalspezifischen Geschäftsmodellkomponenten und Rahmenbedingungen anregt, welche durch das klassische BMC nicht erfasst würden.

Kritisch hinterfragend muss dennoch festgehalten werden, dass die gewonnenen Ergebnisse durch den begrenzten Stichprobenumfang, die immense Komplexität des Geschäftsmodellkonzepts selbst und seiner Umgebungen begrenzt sind. Das vorgeschlagene DC bleibt eine unvollständige Abstraktion, welche nur Teilaspekte dieser immer komplexer werdenden wirtschaftlichen Realität erfassen kann. Dennoch fungiert es als Orientierungshilfe und kann wertvolle Unterstützung bei der digitalspezifischen Konzeption von GM bieten, wovon Studierende und Entrepreneure profitieren.

\section{Zusammenfassung und Ausblick}

Das hier eingeführte DC dient als leitfadengetriebene, strukturierende Visualisierungsgrundlage zur Gestaltung digitaler Geschäftsmodelle. Es berücksichtigt in seinem Aufbau und der Dimensionierung die aus der sich digital-wandelnden hochdynamischen Umwelt erwachsenen Einflussfaktoren auf GM. Damit leistet der empirisch gestützte Forschungsbeitrag eine praxisrelevante Lösung für das Problem der fehlenden Passung des BMC im Digitalisierungskontext. Der Lösungsvorschlag berücksichtigt die verschwimmende Ressourcenintegration zwischen Anbieter und Nachfrager genauso wie die Notwendigkeit der ständigen Anpassung des GMs an die sich ändernden Rahmenbedingungen von Markt, Gesellschaft, Technologien und Regularien.

Der Beitrag bietet Raum für weitere Forschung, im Zuge dessen das DC in verschiedenen Kontexten empirisch evaluiert und ggf. um weitere Bausteine und 
Leitfragen erweitert werden sollte. Eine sich anschließende Diskussion, nachdem weitere Erfahrungen mit der neuen Visualisierungsgrundlage in der Praxis gesammelt worden sind, wäre daher äußerst wünschenswert.

Funding Open Access funding provided by Projekt DEAL.

Open Access Dieser Artikel wird unter der Creative Commons Namensnennung 4.0 International Lizenz veröffentlicht, welche die Nutzung, Vervielfältigung, Bearbeitung, Verbreitung und Wiedergabe in jeglichem Medium und Format erlaubt, sofern Sie den/die ursprünglichen Autor(en) und die Quelle ordnungsgemäß nennen, einen Link zur Creative Commons Lizenz beifügen und angeben, ob Änderungen vorgenommen wurden.

Die in diesem Artikel enthaltenen Bilder und sonstiges Drittmaterial unterliegen ebenfalls der genannten Creative Commons Lizenz, sofern sich aus der Abbildungslegende nichts anderes ergibt. Sofern das betreffende Material nicht unter der genannten Creative Commons Lizenz steht und die betreffende Handlung nicht nach gesetzlichen Vorschriften erlaubt ist, ist für die oben aufgeführten Weiterverwendungen des Materials die Einwilligung des jeweiligen Rechteinhabers einzuholen.

Weitere Details zur Lizenz entnehmen Sie bitte der Lizenzinformation auf http://creativecommons.org/ licenses/by/4.0/deed.de.

\section{Literatur}

Ching H, Fauvel C (2013) Criticisms, variations and experiences with the business model canvas. Eur J Agric Res 1(2):26-37

Coes DH (2014) Critically assessing the strengths and limitations of the business model canvas. Masterarbeit. University of Twente, Nijverdal

Döring N, Bortz J (2016) Forschungsmethoden und Evaluation in den Sozial- und Humanwissenschaften. Gabler, Berlin/Heidelberg

Downs J, Velamuri V (2018) Business model innovation in a knowledge revolution: an evolutory theory perspective. Manag Decis Econ 39:550-562. https://doi.org/10.1002/mde.2926

Hamidian K, Kraijo C (2013) Digitalisierung und Innovation: Planung, Entstehung, Entwicklungsperspektiven. Springer, Wiesbaden

Iansiti M, Lakhani K (2014) Digital ubiquity: how connections, sensors and data are revolutionizing business. Harv Bus Rev 92:91-99

Kreutzer R, Land KH (2016) Digitaler Darwinismus, 2. Aufl. Springer Gabler, Berlin/Köln

Mayring P (2002) Einführung in die qualitative Sozialforschung: Eine Anleitung zu qualitativem Denken, 5. Aufl. Beltz, Weinheim

Meuser M, Nagel U (2009) Experteninterviews - vielfach erprobt, wenig bedacht. In: Bogner A, Littig BE (Hrsg) Das Experteninterview: Theorie, Methode, Anwendung, 3. Aufl. Budrich, Opladen, S 71-93

Osterwalder A, Pigneur Y (2010) Business model generation: a handbook for visionaries, game changers and challengers, 1. Aufl. Wiley, New York

Pfeiffer A (2018) Service-oriented Business-Model-Framework: Die Entwicklung von Geschäftsmodellen in digital transformierenden Ökosystemen am Beispiel der Elektromobilität. Dissertation. RWTH Aachen, Aachen

Reinartz W (2018) Kundenansprache in Zeiten digitaler Transformation. In: Bruhn M, Kirchgeorg M (Hrsg) Marketing Weiterdenken: Zukunftspfade für eine marktorientierte Unternehmensführung. Springer Gabler, Basel/Leipzig, S 124-137

Simmert B, Ebel P, Bretschneider U (2014) Empirische Erkenntnisse zur Nutzung des Business Model Canvas. Working Paper Series der Universität Kassel, Bd. 5, S 2-53

Stenn T (2017) Social entrepreneurship as sustainable development. Springer, Cham

Trimi S, Berbegal-Mirabent J (2012) Business model innovation in entrepreneurship. Int Entrep Manag J 8:449-465. https://doi.org/10.1007/s11365-012-0234-3

Vargo S, Lusch R (2008) Service-dominant logic: continuing the evolution. J Acad Mark Sci 36(1):1-10. https://doi.org/10.1007/s11747-007-0069-6 
Wagner T, Tilly R, Bodenbenner P, Seltiz A, Schoder D (2015) Geschäftsmodellinnovation in der Praxis: Ergebnisse einer Expertenbefragung zum Business Model Canvas und Co. In: Thomas O, Teuteberg F (Hrsg) Proceedings der 12. Internationalen Tagung Wirtschaftsinformatik Osnabrück, S 1298-1312

Wirtz B, Göttel V, Daiser P (2016) Business model innovation: development, concept and future research directions. J Bus Models 4(1):1-28. https://doi.org/10.5278/ojs.jbm.v4i1.1621 